\title{
Bladder leiomyosarcoma in a patient with chronic ketamine abuse: A case report
}

\author{
Dewen Zhong, MD; Feng Yu, MD; Jiande Chen, MD; Chaolu Lin, MD; Huaijing Luo, MD
}

Department of Urology, The First Affiliated Hospital of Fujian Medical University, Longyan, China

Cite as: Can Urol Assoc J 2015;9(7-8):E514-6. http://dx.doi.org/10.5489/cuaj.2662

Published online July 17, 2015.

\section{Abstract}

Bladder leiomyosarcoma is a rare mesenchymal tumour, accounting for less than $0.5 \%$ of all primary bladder malignancies. Adult women of reproductive age have the higher incidence of bladder leiomyosarcoma. Exposure to local pelvic radiotherapy or systemic chemotherapy, especially cyclophosphamide therapy, is also a significant risk factor. We describe a case of a 31-year-old male who developed urinary bladder leiomyosarcoma. The patient had no history of radiotherapy, systemic chemotherapy, or other significant event, except a 5-year history of ketamine abuse. The tumour was found on the left bladder wall and was definitively diagnosed by transurethral resection of the bladder tumour. A partial cystectomy was performed. There are no known reports of urinary bladder leiomyosarcoma associated with chronic ketamine abuse; therefore, we speculate that chronic ketamine abuse may be a factor in the development of this infrequent bladder malignancy.

\section{Introduction}

Benign and malignant mesenchymal tumours of the urinary bladder represent only $0.1 \%$ of bladder cancers in adults, with leiomyosarcomas as the most common type. ${ }^{1}$ The median age of patients with bladder leiomyosarcoma is 52 years and ranges widely from 16 to 83 years..$^{2-4}$ Fewer than 200 cases have been reported. ${ }^{5} \mathrm{~A}$ detailed evaluation of the mechanisms underlying this disease are few, although hereditary retinoblastoma and cyclophosphamide are potential etiological factors. ${ }^{4}$ We describe a case of a young man with urinary bladder leiomyosarcoma, who had no significant medical history except chronic ketamine abuse.

\section{Case presentation}

A 31-year-old man was admitted for macroscopic hematuria and suprapubic discomfort during micturition. He did not experience any frequency, urgency, or lumbago associated with urination. His urinary symptoms worsened after 3 days, and he was subsequently admitted to the Department of Urology. On further questioning, the patient reported that he had been abusing intranasal ketamine for the previous 5 years. At admission, he was alert with a blood pressure of $131 / 96 \mathrm{mmHg}$ and a heart rate of $103 \mathrm{bpm}$. A complete blood count revealed a hemoglobin concentration of $160 \mathrm{~g} / \mathrm{L}$ and a hematocrit of $44.5 \%$. Computed tomography was performed and showed an occupying lesion along the left wall of the urinary bladder (Fig. 1). The ureters and kidneys did not exhibit obvious expansion or hydrops. We initiated emergency treatment, including bladder irrigation with a three-way Foley catheter, intravenous administration of $0.9 \%$ saline, and rest.

Despite treatment, the hematuria worsened, and his face became pale with diaphoresis the following morning. His blood pressure was $89 / 51 \mathrm{mmHg}$, and his heart rate was 115 bpm. Repeat bloodwork showed a hemoglobin concentration of $72 \mathrm{~g} / \mathrm{L}$ and a hematocrit of $18.1 \%$. Emergency treatment for shock was immediately initiated, and 4 units of concentrated red blood cells was administered. Once his vital signs stabilized, a cystoscopy was conducted and revealed a tumour measuring about $5 \times 5 \mathrm{~cm}$ along the left bladder wall. The tumour surface was ulcerated, and a 200$\mathrm{mL}$ blood clot was observed in the bladder. Transurethral resection of the bladder tumour was performed. The patient recovered rapidly postoperatively.

On histopathological examination, a spindle cell tumour with nuclear pleomorphism was identified (Fig. 2). The tumour was diagnosed as a leiomyosarcoma by immunohistochemical staining demonstrating the following results: Ki-67(80\%+), H-Caldesmon(+), CK(-), EMA(-), CD117(-), CD34(-), SMA(-), S-100(-), Desmin(-), DOG-1(-), ALK(-), CD31(-), NF(-), and myosin(-). The patient elected to preserve his bladder and declined chemotherapy. A partial cystectomy without adjuvant therapy was performed, and the patient was discharged. The patient completed a ketamine abuse cessation program. There was no sign of tumour recur- 


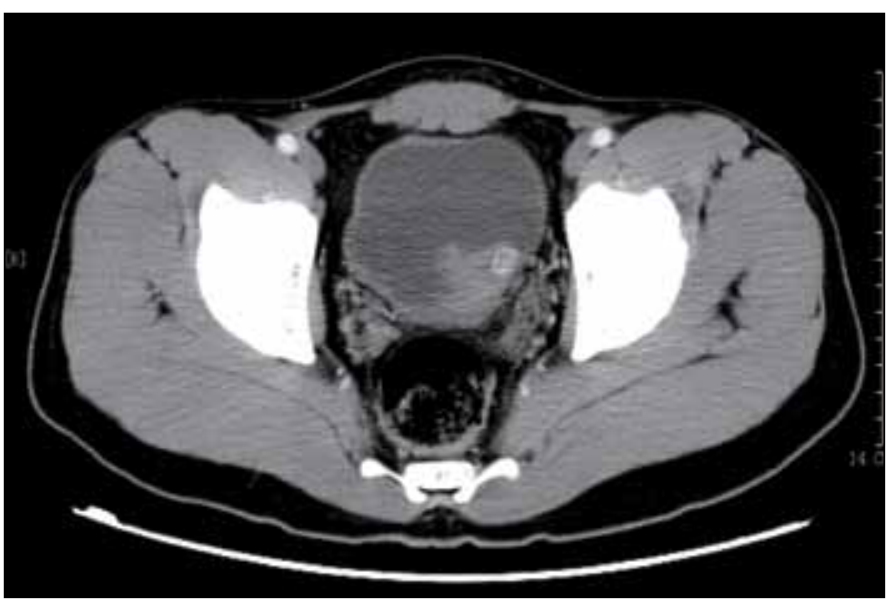

Fig. 1. Computed tomography performed on admission revealed an occupying mass along the left wall of the urinary bladder.

rence or related significant medical findings during 2 years of follow-up.

\section{Discussion}

Bladder leiomyosarcoma is rare and aggressive, and few cases with adequate follow-up have been described. ${ }^{2-4,5}$ Gross hematuria (81\%) is the most common clinical presentation, followed by pollakiuria (28\%), and dysuria $(19 \%){ }^{2}$ Both hereditary retinoblastoma and cyclophosphamide have been identified as potential etiological factors. Fibroblastic proliferation and mucosal telangiectasia have been observed in the bladder in cases of cyclophosphamide cystitis, and both of these atypical mesenchymal alternations have been associated with sarcoma. ${ }^{6,7}$ In hereditary profile retinoblasto$\mathrm{ma}$, some leiomyosarcomas show mutations of the $\mathrm{pRb}$ and p16 genes, which suggests that some individuals may have an inherited susceptibility to leiomyosarcoma development. ${ }^{8}$ However, the present patient did not have retinoblastoma and had not received cyclophosphamide therapy.

The patient had a 5-year history of ketamine abuse. There are no known reports of bladder leiomyosarcoma associated with chronic ketamine abuse. However, ketamine abuse has been associated with other bladder diseases, such as severe ulcerative cystitis. ${ }^{9}$ In 2007, Shahani was the first to describe 9 patients who developed ketamine-associated ulcerative cystitis. This new clinical entity indicates that ketamine abuse can result in bladder dysfunction.

Ketamine (2-[2-chlorophenyl]-2-[methylamino]-cyclohexanone) is a non-competitive $\mathrm{N}$-methyl-D-aspartate (NMDA) receptor antagonist and a derivative of phencyclidine. ${ }^{10}$ Ketamine is a dose-dependent dissociative anesthetic with a short duration of action. It is metabolized by hepatic microsomal enzymes. The major metabolic pathway for ketamine involves $\mathrm{N}$-demethylation to form norketamine, which is in turn hydroxylated to hydroxyl-norketamine.

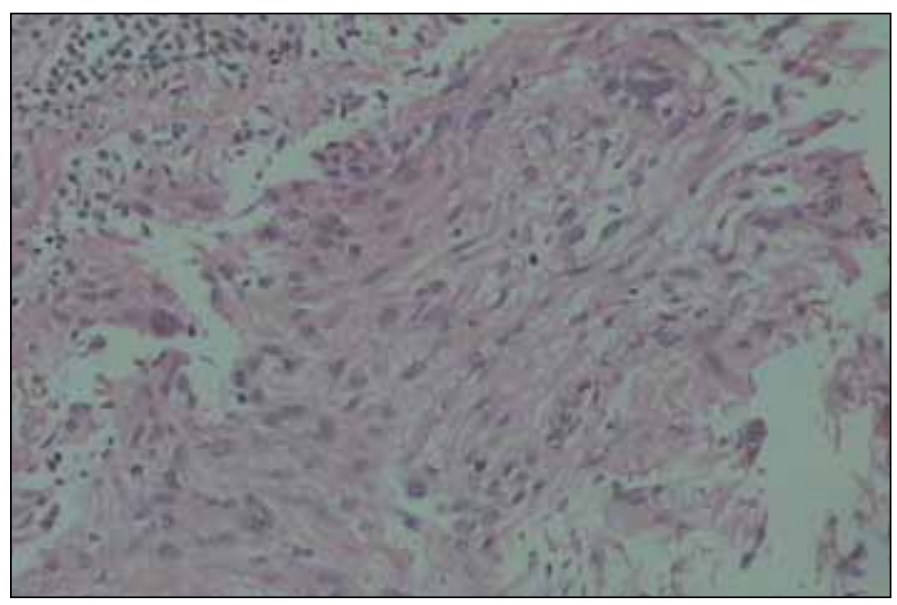

Fig. 2. Histopathologic examination of the biopsied mass reveals spindle cells with nuclear pleomorphism.

These products are conjugated to water-soluble glucuronide derivatives, and $90 \%$ of the metabolites are excreted in the urine. ${ }^{11}$

Chronic exposure to ketamine and its metabolites may be directly toxic to the bladder mucosa and impair the mucosal barrier. As a result, urine, ketamine, and its metabolites can infiltrate into the submucosa, muscular layer, and stroma, which includes mesenchymal cells. This chronic irritation and stimulation may favour pathological changes in the epithelial and mesenchymal cells, ultimately triggering cell transformation and malignant change. The mechanism may be similar to the mechanism of cyclophosphamide and its metabolites, which are known to cause bladder leiomyosarcoma. ${ }^{12}$ However, this potential relationship and hypothesis requires further clinical and scientific investigation.

\section{Conclusion}

This case study describes a basic clinic phenomenon that reminds clinicians, including urologists and psychiatrists, to pay particular attention to the emergence of bladder disease in chronic ketamine abusers, as leiomyosarcoma may result as in the present case. Early clinical intervention or screening tests may be warranted in chronic ketamine abusers, even if they lack any obvious urinary signs or symptoms.

Competing interests: The authors declare no competing financial or personal interests.

This paper has been peer-reviewed.

\section{References}

1. Pedersen-Biergaard J, Jonsson V, Pedersen $M$, et al. Leiomyosarcoma of the urinary bladder after cyclophosphamide. J Clin Oncol 1995;13:532-3. 
2. Yamada T, Nagai S, Kanimoto Y. Rapid progression of a urinary bladder leiomyosarcoma: Report of a case. Case Rep Urol 2011;2011: 532081.

3. Tanguay $C$, Harvey I, Houde $M$, et al. Leiomyosarcoma of urinary bladder following cyclophosphamide therapy: Report of two cases. Mod 2003;16:512-4. http://dx.doi.org/10.1097/01. MP.0000068237.38715.D9

4. Xu YF, Wang GC, Zheng JH, et al. Partial cystectomy: Is it a reliable option for the treatment of bladder leiomyosarcoma? Can Urol Assoc J 2011;5:E1 1-3. http://dx.doi.org/10.1097/01. MP.0000068237.38715.D9

5. Parekh DJ, Jung C, $O^{\prime} C$ Conner J, et al: Leiomyosarcoma in urinary bladder after cyclophosphamide therapy for retinoblastoma and review of bladder sarcomas. Urology 2002;60:164. http://dx.doi.org/10.1016/ S0090-4295(02)01701-6

6. Rowland RG, Eble JN. Bladder leiomyosarcoma and pelvic fibroblastic tumor following cyclophosphamide therapy. J Urol 1983;130:344-6.

7. Chasko SB, Keuhnelian IG, Gutowski WT, III, et al. Spindle cell cancer of bladder during cyclophosphamide therapy for Wegener's granulomatosis. Am I Surg Pathol 1980;4:191-6. http://dx.doi. org/10.1097/00000478-198004000-00011
8. Liang SX, Lakshmanan Y, Woda BA, et al. A high-grade primary leiomyosarcoma of the bladder in a survivor of retinoblastoma. Arch Pathol Lab Med 2001;125:1231-4.

9. Shahani R, Streutker C, Dickson B, et al. Ketamine-associated ulcerative cystitis: A new clinical entity. Urology 2007;69:810-2. http://dx.doi.org/10.1016/j.urology.2007.01.038

10. Corazza, Ornella, Sulaf Assi, et al. From "Special K" to "Special M": The evolution of the recreational use of ketamine and methoxetamine. CNS Neurosci \& Ther 2013;19:454-60. http://dx.doi.org/10.1111/ cns. 12063

11. Lo RS, Krishnamoorthy R, Freeman JG, et al. Cholestasis and biliary dilatation associated with chronic ketamine abuse: A case series. Singapore Med J 2011;52:e52-5.

12. Guarino $M$, Rubino $B$, Ballabio $G$. The role of epithelial-mesenchymal transition in cancer pathology. Pathology 2007;39:305-18. http://dx.doi.org/10.1080/00313020701329914

Correspondence: Dr. Zhong Dewen, Department of Urology, The First Affiliated Hospital of Fujian Medical University, Longyan, China; danxinghu22@126.com 